\title{
Polymer porous thin films obtained by direct spin coating
}

\author{
Alberto Álvarez-Fernández ${ }^{\mathrm{a}}$, Fernando Valdés-Bango ${ }^{\mathrm{b}, \mathrm{c}}$, Raquel Losada-Ambrinos ${ }^{\mathrm{a}}$, José \\ Ignacio Martín $^{\mathrm{b}, \mathrm{c}}$, María Vélez ${ }^{\mathrm{b}, \mathrm{c}}$, José María Alameda ${ }^{\mathrm{b}, \mathrm{c}}$ and Francisco Javier García \\ Alonso $^{\mathrm{a}, \mathrm{c}^{*}}$ \\ a Dpto. Química Orgánica e Inorgánica, Universidad de Oviedo, 33006 Oviedo, Spain. \\ ${ }^{\mathrm{b}}$ Dpto. Física, Universidad de Oviedo, 33007 Oviedo, Spain. \\ ${ }^{\mathrm{c}}$ CINN (CSIC-Universidad de Oviedo), El Entrego, Spain. \\ e-mail: fjga@uniovi.es
}

\begin{abstract}
Films of four different nanostructures, namely, micelles, filled cylinders, ring shaped porous and hollow cylinders, can be prepared easily in a straightforward and simple way by spin coating onto a silicon plate toluene solutions of Poly(styrene-block-4-vinylpyridine) copolymer (PS-b-P4VP) containing formic acid $(\mathrm{H}-\mathrm{COOH})$ of increasing concentration. Despite the enormous progress on preparation of porous polymers, most of them require multiple steps. In this sense, this method represents an effort to obtain not only porous thin films but also other bidimensional nanostructures in a very simple way.
\end{abstract}

Keywords: block-copolymers; self-assembly; natrostructured porous thin films 


\section{Introduction}

Block copolymer (BCP) have been extensively studied in the last decades [1]. They can self-assemble into nanostructured thin films [2]. A vast number of periodic arrays of nanoscale bidimensional structures can be accomplished varying chain lengths, molecular weights, and chemical composition of the homopolymer segments [3], but also by choosing a particular film deposition method (e.g., spin-casting, flow coating, dip coating, zone-casting, electrospray) [4], or subjecting the deposited film to various post-processing techniques, namely, annealing (thermal-, solvent-, vapor-, solvothermal-, light- or laser-promoted photothermal annealing) or alignment (shear- electric- or magnetic alignment) [3], or even preparing previously the substrate by chemical o mechanical modification [4]. The BCP thin films have been used for various applications, such as nanolithography, sensors, microelectronics, nanoreactors, nanoporous films and membranes [5,6].

Polymer nanoporous thin films are frequently obtained using block copolymers as starting materials [7,8]. The reconstruction process is one of the simplest and mildest methods to prepare thin nanoporous films. The procedure consists in introducing a micellar or a cylindrical thin film in the appropriate solvent and after extracting the film, the solvent is evaporated. The process [9] is driven by diffusion of the solvent molecules into the micelle cores that forces them to suffer a plastic deformation which leads first to the glassy corona rupture and then to their overflow over the surface. A similar swelling process occurs with the cylinders driving them to spread out on the surface. After solvent evaporation, a hole is created in the position previously occupied by the micelle core or by the cylinder, however the thin film configuration remains mostly undisturbed because the non swollen matrix is still in the glassy state when exposed to the solvent. Alcohol and water are commonly used as solvents [9], but acetic acid has also been employed as an adequate solvent for preparing porous films starting either from a PS-b-PMM cylindrical films [10] or from micellar PS-b-P2VP films [11,12]. The process is, in general, very fast and it is supposed to occur in two steps. First, the copolymer block located either in the micelle nucleus or in the cylinder swells with the solvent, migrating onto the film surface. Secondly, after evaporation of the solvent, the swelled chains shrink creating the observed pores [9].

A common procedure for the fabrication of highly reproducible polymer thin films is spin coating [13]. The film thickness depends on the spinning velocity, concentration and molecular weight [14]. Because of the rapid evaporation of the solvent, the thermal equilibrium is not reached and, therefore, kinetically controlled nanostructures are obtained [5]. Very recently, the spin-coating process of the formation of PS-b-P2VP thin films has been observed directly by using grazing incidence small-angle X-ray scattering (GISAXS) measurements [15].

In this work, we have found that spin coating toluene solutions of PS-b-P4VP copolymer ( $0.7 \%$ in weight), containing different amounts of formic acid as guest molecules, permits the 
easy preparation of thin films of four different morphologies (micellar, cylindrical, ring shaped porous, and hollow cylindrical). The formation of these nanostructures can be explained taking into account simultaneously the mechanisms of the preparation of the porous films by the reconstruction process [9] and that one observed in the formation of PS-b-P2VP cylindrical thin film by GISAXS [15]. In the way to a complete control of rapid and efficient methods for ordering block-copolymers, the present work represents an effort to obtain nanoporous thin films (and other nanostructures) in a simple way. These results seem particularly interesting considering that there exist defect-tolerant applications of block-copolymer films [16].

\section{Materials and Methods}

\subsection{Materials}

PS-b-P4VP diblock copolymer, with Mn $(\mathrm{PS})=47.0 \mathrm{~kg} / \mathrm{mol}$ and $\mathrm{Mn}(\mathrm{P} 4 \mathrm{VP})=25.0$ $\mathrm{kg} / \mathrm{mol}$, was purchased from Polymer Source Inc. and used as received. This copolymer corresponds to the hexagonal packed P4VP cylinders phase because of the relationship between Polystyrene volume fraction and Poly(4-Vinylpyridine) volume fraction [17,18]. Formic acid (puriss. p.a., ACS reagent, reag. Ph. Eur., $\geq 98 \%$ ), toluene (dry, 99.8\%) and ethanol (absolute, 99.5) were purchased from Sigma-Aldrich and Merck respectively, and used without further purification. Silicon wafers $\left(\begin{array}{lll}1 & 0 & 0\end{array}\right)$ were purchased from CrysTec $\mathrm{GmbH}$ and cut to appropriate dimensions (approximately $1 \times 1 \mathrm{~cm}^{2}$ ). Silicon substrates were successively cleaned in acetone and Milli-Q water in an ultrasonic bath, and dried under nitrogen flow. $50 \mathrm{~nm}$ thick silicon nitride membranes prepared for TEM measurements were purchased from Agar Scientific.

\subsection{Sample preparation}

The starting copolymer solutions were prepared following the subsequent methodology.

Solutions of PS-b-P4VP (0.7 wt. \%) with the appropriate amount of formic acid for the desired $\mathrm{HCOOH} / \mathrm{P} 4 \mathrm{VP}$ molar ratios were prepared in dry toluene and stirred overnight in closed vials on a heating plate at ca. $70{ }^{\circ} \mathrm{C}$. Then they were filtered through $0.45 \mu \mathrm{n}$ filters (VWR). The copolymer solutions were spin-coated at $4000 \mathrm{rpm}$ of rotational speed and at $2000 \mathrm{rpm} / \mathrm{s}$ of rotational acceleration, using a Spinner SPIN 150 (SPS), onto silicon wafers or silicon nitride substrates to prepare the copolymer film. Identical results are obtained using silicon or silicon nitride. To ensure reproducibility, every experiment was carried out at least four times with exactly the same results.

\subsection{Atomic Force Microscopy (AFM)}

Topography AFM images were obtained in contact mode using a Scanning Probe Microscope controlled by a Nanotec controller (Nanotec S.L.), operated under an ambient atmosphere. $\mathrm{Si}_{3} \mathrm{~N}_{4}$ tips with $15 \mathrm{~nm}$ radius and enhanced reflectivity through $\mathrm{Au} / \mathrm{Cr}$ coating were 
purchased from Olympus. Average intermicellar spacings were obtained from the relevant period in Fast Fourier Transforms of the real space topography images. Average micellar diameters were obtained from analysis of real space topography images with the "flooding" tool of WSxM software.

\subsection{Infrared spectra}

The infrared spectra (IR) were recorded with a Perkin Elmer Paragon 1000 FT-IR spectrometer.

\subsection{Transmission Electron Microscopy (TEM)}

TEM images were obtained with a MET JEOL-2000 EX-II on films deposited on $\mathrm{Si}_{3} \mathrm{~N}_{4}$ TEM membranes and consecutively tinctured through exposure to $I_{2}$ vapors in a closed vessel for $24 \mathrm{~h}$ due to its affinity to pyridine domains.

\subsection{Ellipsometry}

In order to determine the film thickness, spectroscopic ellipsometry measurements have been obtained with a Horiba UVISEL variable angle spectroscopic ellipsometry (VASE) in reflection, on the spectral range from 450 to $1900 \mathrm{~nm}$. The spot size was less than $1 \mathrm{~mm}$. The angle of incidence was varied in the range of $60^{\circ}$ to $90^{\circ}$.

\subsection{Dynamic Light Scattering (DLS)}

Size distributions of the PS-b-P4VP (0.7 wt. \%) toluene solutions, with different $\mathrm{HCOOH} / \mathrm{P} 4 \mathrm{VP}$ ratios $(0 / 1 ; 3 / 1 ; 5 / 1$; and $10 / 1)$ were measured with a Malvern High Performance Particle Sizer (HPPS). Each measurement was run 5 times.

\section{Results and Discussion}

\subsection{Study of the polymer-host molecule interaction in solution}

Toluene solutions of PS-b-P4VP (0.7 wt. \%) with different molar ratios HCOOH/P4VP were studied by DLS. As toluene is a good solvent for PS, but non-solvent for P4VP, spherical micellar structures are formed in solution in all the cases, consisting in a PS shell and a P4VP nucleus with a certain number of formic acid molecules linked to the pyridine ring since organic acids have been frequently used as host molecules bonded to pyridine ring in the PS-b-P4VP copolymers $[19,20]$. DLS measures show a gradual increment of the micellar diameter related with the gradual increment of the $\mathrm{HCOOH} / \mathrm{P} 4 \mathrm{VP}$ ratios in the solutions. Thus, micellar diameter is increased from $81 \mathrm{~nm}$ (Figure 1, curve A) when the toluene contains merely PS-b-P4VP, to $99 \mathrm{~nm}$ (Figure 1, curve B) when the solution includes formic acid in a HCOOH/P4VP 1/1 ratio. 

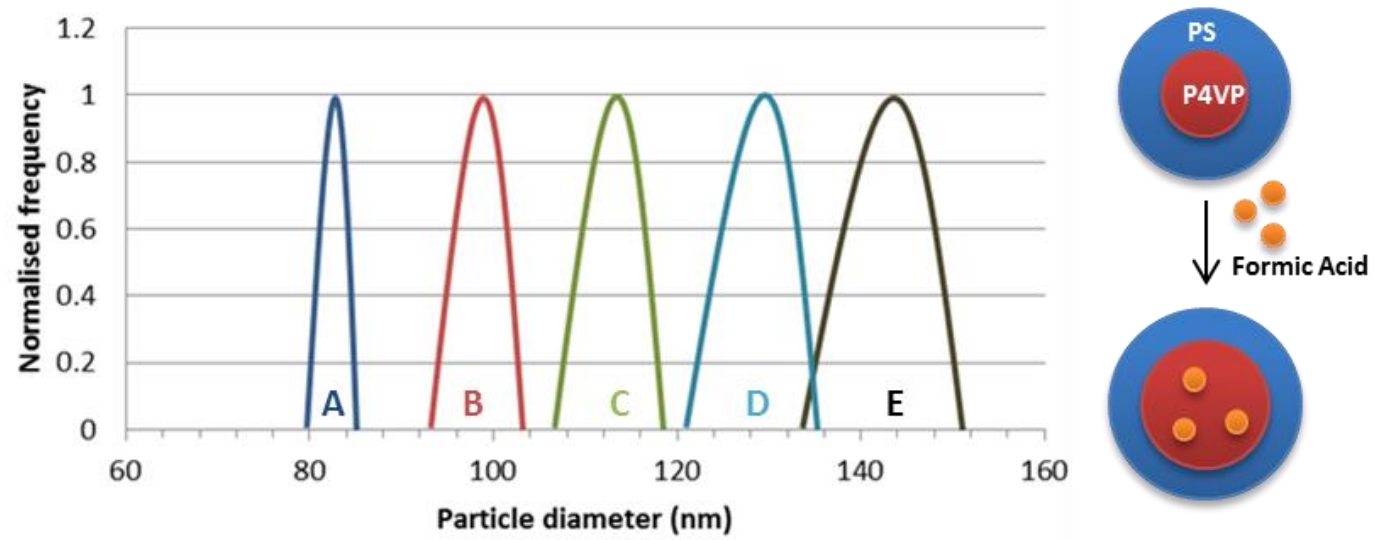

Figure 1. DLS size distributions for toluene solution of (PS-b-P4VP) $(0.7 w t \%)$ containing formic acid at different HCOOH/P4VP molar ratios: 0/1(A), 1/1 (B), 3/1 (C), 5/1 (D) and 10/1 $(E)$ and schematic representation of micellar growth with the formic acid inclusion.

Micellar size continues increasing with the incorporation of more formic acid to the solution, $117 \mathrm{~nm}$ (Figure 1, curve C) for 3/1 HCOOH/P4VP ratio and $129 \mathrm{~nm}$ (Figure 1, curve D) and $143 \mathrm{~nm}$ (Figure 1, curve $\mathrm{E}$ ) for 5/1 and 10/1 ratio.

The nature of the bond between the guest molecule $(\mathrm{HCOOH})$ and the block copolymer could be understood taking into account the infrared spectra collected in Figure 2 corresponding to samples obtained after evaporating in vacuo an aliquot of the starting toluene solutions of PS-b-P4VP (0.7 wt \%) with the appropriate $\mathrm{HCOOH} / \mathrm{P} 4 \mathrm{VP}$ molar ratio (0/1, A; 1/1, B; 3/1, C; 5/1, D; and 10/1; E) onto a $\mathrm{KBr}$ disk.

Many $\mathrm{HCOOH}$ molecules should be $\mathrm{H}$-bonded to the nitrogen atoms of the pyridine groups, since the bands at 1596 and $1414 \mathrm{~cm}^{-1}$ corresponding free pyridine in PS-b-P4VP (Figure 2A) appear a little bit shifted in all the spectra (at 1599 and $1416 \mathrm{~cm}^{-1}$ in B, at 1601 and $1418 \mathrm{~cm}^{-1}$ in $\mathrm{C}$, and at 1603 and $1419 \mathrm{~cm}^{-1}$ in D and in E), just as it is expected to occur when the pyridine is H-bonded to a carboxylic acid [21]. Other broad bands at 2580 and $1946 \mathrm{~cm}^{-1}$ present in the spectra C, D and E of Figure 2 are also indicative of a strong H-bond between carboxylic acid and pyridine [22] although they are almost imperceptible in Figure 2B. Furthermore, the signals at 1755 , sh, and $1714, \mathrm{~s}_{\mathrm{cm}} \mathrm{cm}^{-1}$ should be assigned to the $\mathrm{C}=\mathrm{O}$ stretching band of a formic acid, H-bonded to a pyridine group [23]. Curiously, a rise in the $\mathrm{HCOOH}$ proportion in the starting solution leads to a greater shift in the frequencies of certain $\mathrm{H}$-bonded pyridine bands (those who moved from 1599 and $1416 \mathrm{~cm}^{-1}$ to higher wavenumber) and to an increase of intensity in the other bands that corroborate the H-bond between pyridine and $\mathrm{HCOOH}$, namely those at 2580, 1946 and $1713 \mathrm{~cm}^{-1}$. 

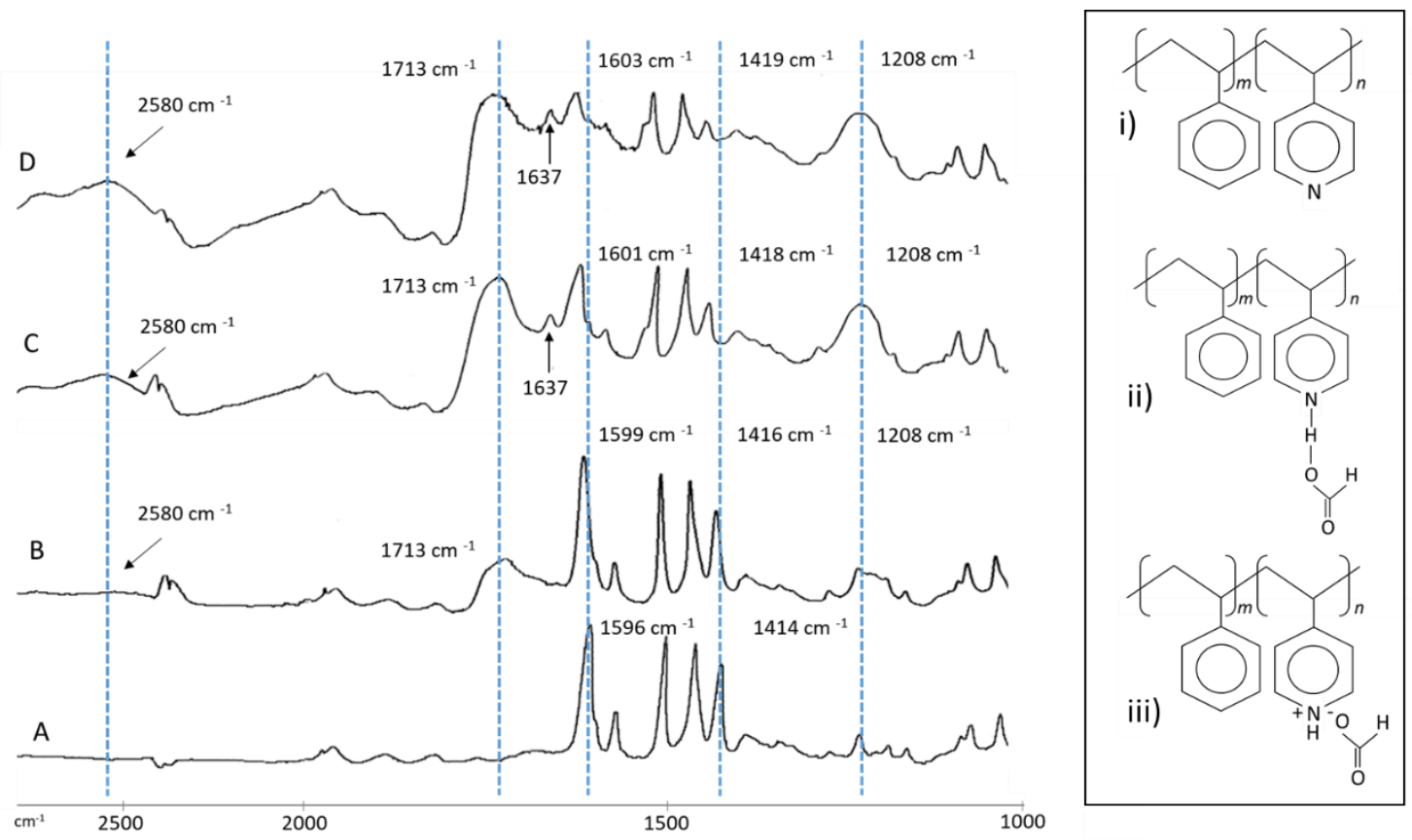

Figure 2. Infrared spectra (from 3000 to $900 \mathrm{~cm}$-1) of samples obtained evaporating in vacuum toluene solutions of PS-b-P4VP $(0.7 w t \%)$ containing formic acid in a HCOOH/P4VP molar ratio: A) $0 / 1$; B) $1 / 1$; C) $3 / 1$; D) $5 / 1$; E) 10/1. On the right side, schematic representation of the chemical structures of pure PS-b-P4VP (i) and the two types of interaction with the formic acid observed in the IR-spectra, H-bonded (ii) or ionic bonded (iii).

However, at higher $\mathrm{HCOOH}$ concentration, the formic acid is able to protonate some pyridine rings as it is evidenced by the presence of an absorption band at $1630 \mathrm{~cm}^{-1}$ (clearly perceptible in the spectra C, D and E of Figure 2) due to the presence of pyridinium ions [24]. Besides, there exists a band at $1208 \mathrm{~cm}^{-1}$ whose intensity has increased from $3 \mathrm{~B}$ to $3 \mathrm{E}$. It may be tentatively assigned to a $\mathrm{C}-\mathrm{O}$ stretching vibration for an $\mathrm{H}$-bonded formic acid since a very strong peak appears at $1218 \mathrm{~cm}^{-1}$ in the infrared spectrum of the dimeric $\mathrm{HCOOH}$ [25].

Thus, the IR characterization clearly shows that, as the proportion of formic acid in the solution increases, there is a larger amount of $\mathrm{HCOOH}$ bonded to P4VP cores. This is reflected in the steady increase of the micelles diameter in solution from $81 \mathrm{~nm}$ for pure PS-b-P4VP to $143 \mathrm{~nm}$ for those containing a $\mathrm{HCOOH} / \mathrm{P} 4 \mathrm{VP}$ in a 10/1 ratio, and, in some way, in the thickness of the obtained films by spin coating from $35 \mathrm{~nm}$ for PS-b-P4VP until to $41 \mathrm{~nm}$ in the last case.

\subsection{Morphological study of polymer-host molecule thin films obtained by spin coating}

Toluene solutions of PS-b- P4VP (0.7 wt. \%) with different $\mathrm{HCOOH} / \mathrm{P} 4 \mathrm{VP}$ ratios were spin-coated onto cleaned silicon substrates. Figure 3 shows the AFM and TEM characterization of the monolayer film (38 nm thick) obtained spin-coating a toluene solution of PS-b-P4VP (0.7 wt \%) containing formic acid in a HCOOH/P4VP 1/1 molar ratio. The AFM micrograph (Figure 3A) exhibits a dimple type structure, suggesting a micellar morphology (very similar, for 
instance, to the morphology of micellar films in [26]) with the micelles consisting in a P4VPHOOCH nucleus and a PS shell as it is shown in Scheme 1(A). This is corroborated by the TEM image (Figure 3B) that shows a pattern of black spots corresponding to the P4VP nuclei of the micelles since the film was exposed to iodine vapor to darken the position of the pyridine groups. A typical height profile, shown in Figure 3C, allows us to estimate the size of the micelles around $90 \mathrm{~nm}$, which is greater than that of the micelles obtained in the same way from a pure PS-b-P4VP toluene solution $(0.7 \mathrm{wt} \%)(58 \mathrm{~nm}$, not shown). This increment in micelle size could be anticipated by the incorporation of the guest molecules into the P4VP nucleus shown in the DLS and IR characterization.
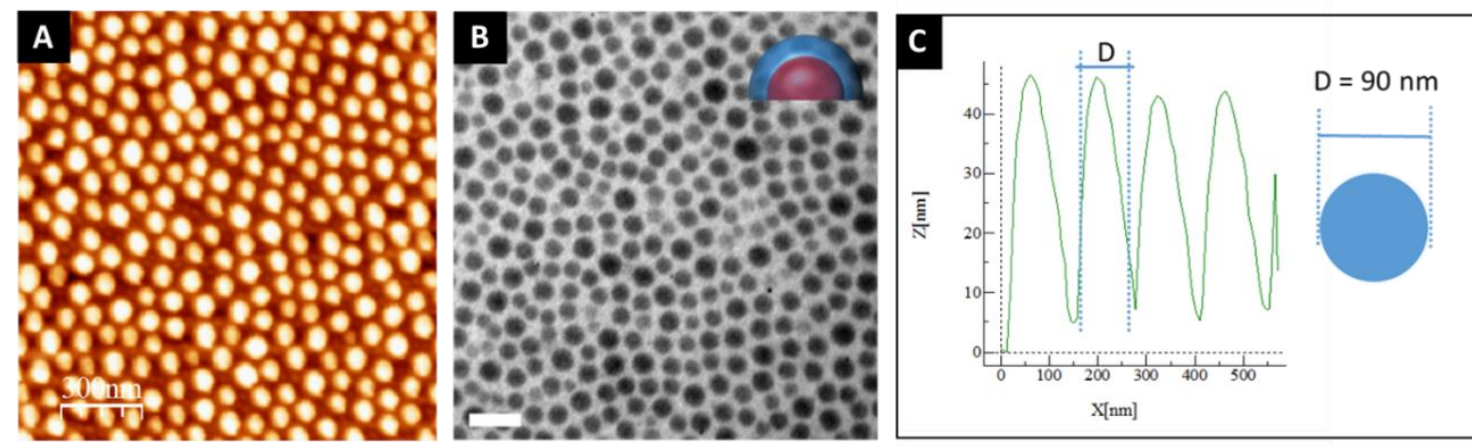

Figure 3. Morphological characterization of the film obtained by spin-coating a toluene solution of (PS-b-P4VP) (0.7 wt \%) containing formic acid at 1/1 HCOOH/P4VP molar ratio: (A) AFM height image; (B) TEM micrographs. Scale bar is $100 \mathrm{~nm}$. Upper right inset depicts the proposed internal structure. (C) Detailed AFM sectional profile.

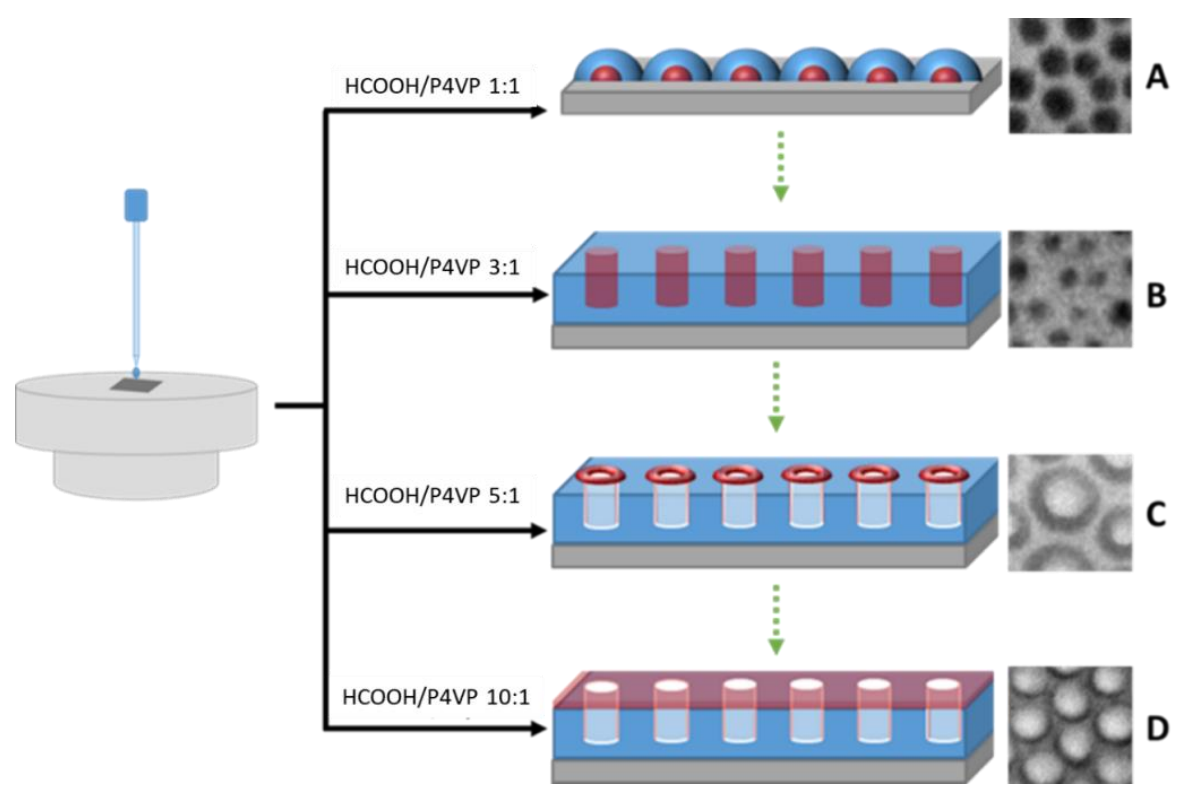

Scheme 1. Representative scheme of the proposed morphologies. Red regions indicate those of P4VP phases, blue ones correspond to PS phases while the white sections are empty. For comparison TEM micrographs are attached, corresponding to the films obtained by spincoating toluene solutions of $(P S-b-P 4 V P)(0.7 w t \%)$ containing formic acid at different HCOOH/P4VP molar ratios: $1 / 1(A), 3 / 1(B), 5 / 1(C)$ and 10/1 (D)). 
The AFM micrograph of the monolayer film (37 nm thick) prepared by spin coating a toluene solution of PS-b-P4VP (0.7 wt. \%) with a $\mathrm{HCOOH/P4VP} \mathrm{3/1} \mathrm{molar} \mathrm{ratio,} \mathrm{onto} \mathrm{a} \mathrm{silicon}$ plate, is shown in Figure 4A. It presents a relatively flat surface with a set of small depressions approximately $6 \mathrm{~nm}$ deep and $80 \mathrm{~nm}$ wide, as it is indicated by the sectional profile (Figure 4C). These low depressions are a typical feature of cylindrical films [27]. However, due to the finite AFM tip radius and tip aspect ratio, AFM characterization is not enough to discriminate between slightly depressed filled cylinders and empty nanopores for these small feature size and the combination with TEM measurements is crucial to obtain a clear image of sample morphology. The TEM image in Figure 4B shows a pattern of dark spots corresponding to the round depressions in the AFM image indicating that they correspond to P4VP cylindrical phases embedded in a PS matrix. For a side and top view image of this morphology see picture B in Scheme 1.
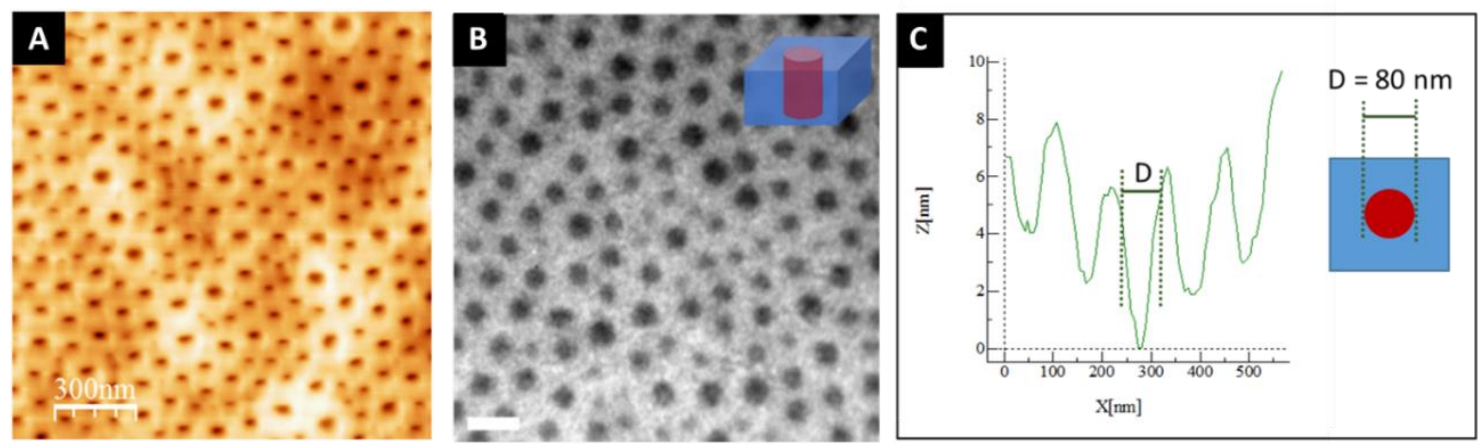

Figure 4. Morphological characterization of the film obtained by spin-coating a toluene solution of (PS-b-P4VP) (0.7 wt \%) containing formic acid at 3/1 HCOOH/P4VP molar ratio: (A) AFM height image; (B) TEM micrographs. Scale bar is $100 \mathrm{~nm}$. Upper right inset depicts the proposed internal structure. (C) Detailed AFM sectional profile.

When a toluene solution of PS-b-P4VP (0.7 wt \%) containing formic acid in a $\mathrm{HCOOH} / \mathrm{P} 4 \mathrm{VP} 5 / 1$ molar ratio is spin coated onto a silicon plate, the resulting $39 \mathrm{~nm}$ thick monolayer film consists in a regular distribution of nanorings as it is shown in the AFM micrograph of Figure 5A (very similar to the nanorings obtained in [28]). The corresponding AFM sectional profile (Figure 5C) indicates that the rings have an external diameter around 130 $\mathrm{nm}$ and an internal diameter circa $70 \mathrm{~nm}$. Once again, the related TEM micrograph (see Figure 5B) is essential to obtain information of the location of pyridine groups in the film: now, the TEM image shows a pattern of dark rings that correspond to the elevated rings in the AFM micrograph. This suggests that the pyridine groups are neither inside nor outside the nanorings but, rather, that the P4VP phases occupy some circular space around them on the surface and, eventually, the inside walls of hollow cylinders too. This annular morphology has been depicted in image $\mathrm{C}$ of scheme 1 . 

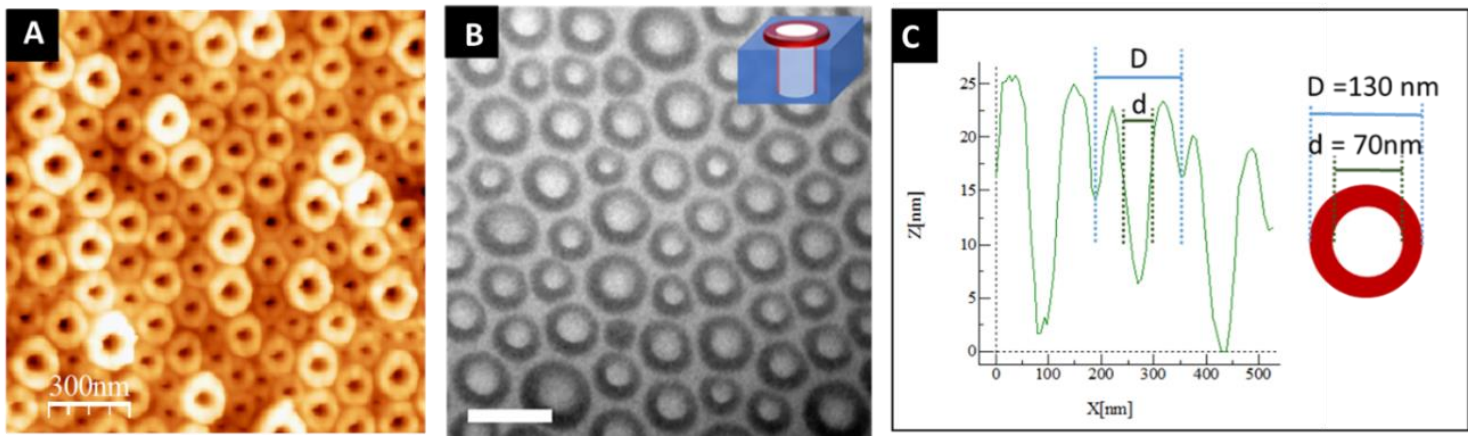

Figure 5. Morphological characterization of the film obtained by spin-coating a toluene solution of (PS-b-P4VP) (0.7 wt \%) containing formic acid at 5/1 HCOOH/P4VP molar ratio: (A) AFM height image; (B) TEM micrographs. Scale bar is $100 \mathrm{~nm}$. Upper right inset depicts the proposed internal structure. $(C)$ Detailed AFM sectional profile.

Finally, the AFM and TEM micrographs (Figure 6) of the $41 \mathrm{~nm}$ thick monolayer film obtained by spin-coating onto a silicon plate a toluene solution of PS-b-P4VP (0.7 wt \%) containing formic acid in a $\mathrm{HCOOH} / \mathrm{P} 4 \mathrm{VP}$ 10/1 molar ratio suggest that its nanostructure consists in hollow cylinders hexagonally distributed inside a matrix of copolymers. The AFM image (Figure 6A), shows a pattern of small depressions within a flat surface. However, the corresponding TEM picture (Figure 6B) suggests that these depressions are empty: the white spots in the middle of the black circles in Figure 6B not only indicate that there is not any pyridine derivative in this area, but also imply that they correspond to empty cylinders, as far as they are in a region previously occupied by the vinylpyridine phase. Now, the vinylpyridine fraction seems to be spread over the PS phases. Thus, the final film morphology (picture D of Scheme 1) consists of hollow cylinders with a P4VP/formic acid phase covering the whole surface of the PS phase although some of it could eventually cover also the inside walls of the hollow cylinders (see, for comparison, the nanoporous morphology in [29]).
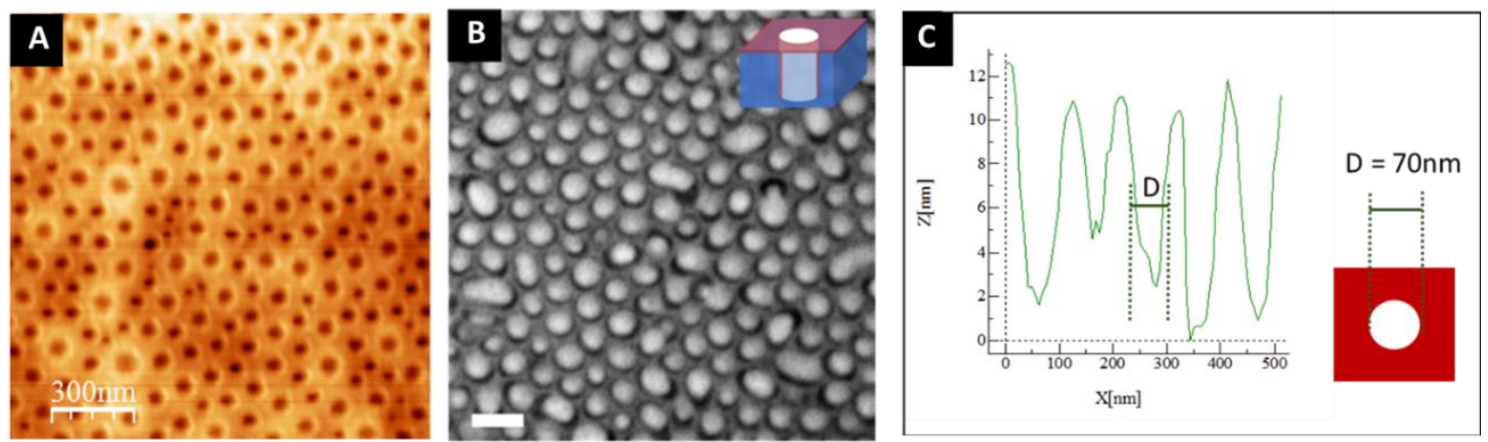

Figure 6. Morphological characterization of the film obtained by spin-coating a toluene solution of (PS-b-P4VP) (0.7 wt \%) containing formic acid at 10/1 HCOOH/P4VP molar ratio: (A) AFM height image; (B) TEM micrographs. Scale bar is $100 \mathrm{~nm}$. Upper right inset depicts the proposed internal structure. (C) Detailed AFM sectional profile. 
Since the polymers form spherical micelles in solution, the formation of a monolayer thin film with micellar morphology (A in scheme 1) is more or less straight-forward. On the contrary, the development of the other three observed nanostructures needs a more sophisticated mechanism. Fortunately, their patterns can be satisfactorily explained assuming that the process of film making is similar to that observed in situ by GISAXS measurements when a toluene solution of an asymmetric PS-b-P2VP polymer was spin-coated on a silicon wafer [15], and taking into account the generally accepted mechanism for the preparation of porous films by the reconstruction process [9].

At the beginning of the spin coating process leading to the formation of vertical cylinders, B in scheme 1, the solution is spread over the substrate while a fast solvent evaporation takes place simultaneously. According to [15], cylinders form because evaporation stops first in the P4VP areas and during the latter evaporation of the PS region, bulk microdomains coalesce forming cylindrical structures perpendicular to the surface. These grow up in the same direction because the residual solvent has evaporated from both components throughout the thickness of the film. Finally, the evaporation is complete and the mobility of the microdomains is suppressed [15].

However, if the pyridine moieties contain enough formic acid, during the evaporation of the last residues of the solvent they should spread out from the initially formed cylinders [9] until they cover only the surrounds of the original cylindrical microdomain, leaving a hole inside, that is, giving rise to the ring shaped porous nanostructure ( $\mathrm{C}$ in Scheme 1). In the same way, when the HCOOH/P4VP ratio is 10/1 this process will continue and, finally, the swollen P4VP phase would cover the whole PS surface enhancing the hollow cylinder (D in Scheme 1).

\section{Conclusions}

Films of four different morphologies have been prepared by spin coating toluene solutions of PS-b-P4VP $(0,7 \mathrm{wt} \%)$ containing increasing amounts of formic acid onto a silicon wafer (A, micellar; B, filled cylinder; C, ring shaped porous; D, hollow cylinders). The formation of these nanostructures is mediated by the bonding of the formic acid molecules to the nitrogen atoms of the P4VP groups through $\mathrm{H}$-bonds, a process that is enhanced as the proportion of acid in the solution is increased. It produces a steady increase of the original micelles diameter what results in the different observed morphologies during solvent evaporation. It supposes another step in the increasing total control of nanostructured blockcopolymer monolayer films in a simple way.

\section{Acknowledgements}

Work supported by Spanish MINECO [FIS2013-45469 and FIS2016-76058 (AEI/FEDER,EU)]. 


\section{References}

[1] C. M. Bates, and Frank S. Bates, Macromolecules (2017) 50, 3

[2] P. W. Majewski, and K. G. Yager, J. Phys. Condens. Matter (2016) 28, 403002

[3] C. Jin, B. C. Olsen, E. J. Luber, and J. M. Buriak, Chem. Mater. (2017) 29, 176 and references therein.

[4] C. K. Shelton, and T. H. Epps III, Polymer (2016) 105, 545 and references therein.

[5] D. Posselt, J.Zhang, D-M. Smilgies, A. V. Berezkin, I. I. Potemkin, and C.M. Papadakis, Progress in Polymer Science (2017) 66, 80

[6] S. Kim, H. S. Wang, Y. Choe, S-H. Choi and J. Bang, Polymer Journal (2016) 48, 333

[7] D. Wu, F. Xu, B. Sun, R. Fu, H. He, and K. Matyjaszewski, Chem. Rev. (2012) 112, 3959

[8] Y. Li, Y. Xu, S. Cao, Y. Zhao, T. Qu, T. Iyoda, and A. Chen, Macromol. Rapid Commun. 2017, DOI: $10.1002 /$ marc.201600662

[9] Y. Wang and F. Li, Adv. Mater. (2011) 23, 2134

[10] T. Xu, J. Stevens, J.A. Villa, J. T. Goldbach, K.W. Guarini, C.T. Black, C.J. Hawker and T. P. Russell, Adv. Funct. Mater. (2003) 13, 698

[11] X. Li, S. Tian, Y. Ping, D. H. Kim and W. Knoll, Langmuir (2005) 21, 9393

[12] Y. Wang, M. Becker, L. Wang, J. Liu, R. Scholz, J. Peng, U. Gösele, S. Christiansen, D. H. Kim, and M. Steinhart, Nano Lett. (2009) 9, 2384

[13] K. Norman, A. Ghanbari-Siahkali and N. B. Larsen, Annu. Rep. Prog. Chem., Sect. C, (2005) 101,174

[14] D. W. Schubert, and T. Dunkel, Mat. Res. Innovat. (2003) 7, 314

[15] H. Ogawa, M. Takenaka, T. Miyazaki, A. Fujiwara, B. Lee, K. Shimokita, E. Nishibori, and M. Takata, Macromolecules (2016) 49, 3471

[16] R. Lundy, S. P. Flynn, C. Cummins, S. M. Kelleher, M. N. Collins, E. Dalton, S. Daniels, M. A. Morris and R. Enright, Phys. Chem. Chem. Phys (2017) DOI: 10.1039/c6cp07633e

[17] M.F. Schulz, A. K. Khandpur, F. S. Bates, K. Almdal, K. Mortensen, D. A. Hajduk and S. M. Gruner, Macromolecules (1996) 29, 2857

[18] W. Zha, C. D. Han, D. H. Lee, S. H. Han, J. K. Kim, J. H. Kang and C. Park, C. Macromolecules (2007) 40, 2109

[19] S-H. Yun, S. Il Yoo, J. C. Jung, W.-C. Zin, and B.-H. Sohn, Chem. Mater. (2006) 18, 5646

[20] D. Chen and M. Jiang, Acc. Chem. Res. (2005) 38, 494

[21] J. Ruokolainen, G. ten Brinke, O. Ikkala, M. Torkkeli and R. Serimaa, Macromolecules (1996) 29,3409

[22] S. Jiang, A. Göpfert and V. Abetz, Macromolecules (2003) 36, 6171

N. V. Drichko, G. Y. Kerenskaia and V. M. Schreiber, J. Mol. Struct. (1999) 477, 127

[24] M. Tiitu, M. Torkkeli, R. Serimaa, T. Mäkelä and O. T. Ikkala, Solid State Ionics (2005)

176,1291 
[25] Y. T. Chang, Y. Yamaguchi, W. H. Miller, H. F. Schaefer III, J. Am. Chem. Soc. (1987) 109,7245

[26] R. Lundy, S. P. Flynn, C. Cummins, S. M. Kelleher, M. N. Collins, E. Dalton, S. Daniels, M. A. Morris and R. Enright, Phys. Chem. Chem. Phys. (2017) 19, 2805

[27] S. Park, B. Kim, J-Y. Wang and Thomas P. Russell, Adv. Mater. (2008) 20, 681

[28] Y. Liu, Y. Gong, L. He, B. Xie, X. Chen, M. Han and G. Wang, Nanoscale (2010) 2, 2065

[29] A. Laforgue, C. G. Bazuin, and R. E. Prud'homme, Macromolecules (2006) 39, 6473 